\title{
AN APPLICATION OF THE SAKAI'S THEOREM TO THE CHARACTERIZATION OF $H^{*}$-ALGEBRAS
}

\author{
BORUT ZALAR \\ University of I.jul,ljana \\ Institute of Mathematics. Jadranuka 19 \\ 61000 I.jubljana, SI.OVT.NI.J.I \\ (Received May 11, 1993)
}

\begin{abstract}
$\Lambda B S T R \Lambda C T$. The well-known Sakai's theorem, which states that every derivation acting on a von Neumann algebra is inner, is used to ol,t ain a new elegant proof of the Saworot now's characterization theorem for associative $I I^{*}$-algebras ria two-siderl $I I^{*}$-algehras. This proof completely avoids structure theory.
\end{abstract}

KEY WORDS AND PIIRASCS. $I I^{*}$-algol,ra, involution, automorphism, derivation, centralizer, von Neumann algebra.

1991 AMS SUB.JECT CLASSIFICATION CODES. 16K1T, 16II20, 46L10, 46L.57.

\section{PRELIMINARIES.}

One of the important (and very derp) results in the therory of von Neumann algebras states that all derivations of von Ncumann algebras are inner. This result was established by Sakai in [7].

SAKAIS TIIEOREM. Let $\mathcal{R}$ be a von Neumann alyrbra and $D$ deriration of $\mathcal{R}$. Then there exists some $a \in \mathcal{R}$ such that $D(x)=a x-x a$ for all $x \in \mathcal{R}$.

This result has many applications and we present another one. We show how the Sakai's theorem can be used to give a new proof of the characterization of $H^{*}$-algcbras in the terms of two-sided $H^{*}$-algebras. This characterization was estahlished by Saworotnov in [8] who described the structure of two-sided $I^{*}$-algebras and then compare it with the strurture of (one-sided) $H^{*}$ algebras obtained by Ambrose in [1].

The theory of associative $I^{*}$-algebras started in 1915 when .1 mbrose introduced this concept 
in order to obtain an abstrart characterisation of the Hillert Se humidt operators. Later various generalizations appeared such as two-bided $/ I^{*}$-algeblas. complemented algebras and Ililbert modules. We refer to [8], [9], [10], [11] and [1:2]. Nonaseoriative $I^{*}$-algebras were also studied. Some recent papers on this subject are [3]. [1]. [.]. [6] and [1:3]. Ambrose int rodured the following

DEFINITION. A complex algebra $\mathcal{A}$ which in aloo a Hillect space with the inner product $\langle$, and an algebra involution $*: \mathcal{A} \longrightarrow \mathcal{A}$ is called an $I^{*}$-alyr bra if the following $I^{*}$-conditions

$$
\langle r y, z\rangle=\left\langle r, z y^{*}\right\rangle=\left\langle\eta, r^{*} z\right\rangle
$$

are satisfied for all $x, y . z \in \mathcal{A}$. $\Lambda$ morkl for such algebra is the algebra $\mathbf{U S}(\mathcal{H})$ of all HilbertSchmidt operators acting on a IIilleet spare $\mathcal{H}$ together with the inner product $\langle T, S\rangle=\operatorname{trace}\left(T S^{*}\right)$.

If we take a look at the $I I^{*}$-conditions, we can ask what happens if the algelora $\mathcal{A}$ would have two involutions: one left and one right. This leaded to the following:

DFFINITION. $A$ complex algebra $\mathcal{A}$ which is aloo a Hillorert space with an inner product $\langle$, is called a two-sided $I I^{*}$-algrbra if for crery $r \in \mathcal{A}$ there cvint clements $r^{r} . x^{l} \in \mathcal{A}$ called a right and a left adjoint of the element $x$ such that

$$
\langle r y, z\rangle=\left\langle y, x^{l} z\right\rangle, \quad\langle y r, z\rangle=\left\langle y, z x^{r}\right\rangle
$$

holds for all $y, z \in \mathcal{A}$.

A model for a two-sided $I^{*}$-algebra which is not an $I^{*}$-algebra can be found in [8]. However Saworotnow proved that two-sided $I^{*}$-algebras are very close to $I^{*}$-algebra. Explicitly he proved

TIIEOREN. Let $(\mathcal{A},(\rangle, I, r$,$) br a two-sided asencululire I^{*}$-algebra wilh zero annihilator. There exists another inner product $\langle,\rangle_{1}$ on $\mathcal{A}$ such that $\left(\mathcal{A} .\langle,\rangle_{1}, r\right)$ becomes an $H^{*}$-algebra.

Before we start our new proof of this result we introduce the following notation for the annihilators of $\mathcal{A}$ :

left annililator: $\operatorname{Lann}(\mathcal{A})=\{r \in \mathcal{A}: r \mathcal{A}=(0)\}$,

right annihilator : $\operatorname{Rann}(\mathcal{A})=\{r \in \mathcal{A}: \mathcal{A} r=(0)\}$.

annihilator: $\Lambda \mathrm{nn}(\mathcal{A})=\operatorname{Lann}(\mathcal{A}) \cap \operatorname{Rann}(\mathcal{A})$.

\section{RESULTS.}

First we shall collect some simple olservations on two-viled $I^{*}$-algebras in the following:

LEMIMA. Let $(\mathcal{A},\langle\rangle, l, r$,$) br a luro-vided associulire I^{*}$-algebra wilh zrro anmihilator. Then the following holds: 
(i) $\operatorname{Lann}(\mathcal{A})=\operatorname{Rann}(\mathcal{A})=(0)$.

(ii) A left and right adjomt of an!y dement are unnune and the mappmings $x \longmapsto x^{r}, x \longmapsto x^{l}$ are both algcbranc involutions.

(iii) The product of $\mathcal{A}$ is contmuous.

(iv) $\mathcal{A}^{2}$ is dense in $\mathcal{A}$.

(v) Bolh involutions $x \longmapsto x^{r}$ and $x \mapsto x^{l}$ arr conlımuous.

(vi) $\langle x, y\rangle=\left\langle y^{r}, x^{l}\right\rangle=\left\langle y^{l}, x^{r}\right\rangle$ holds: for all $x, y \in \mathcal{A}$.

Proof. (i) Suppose that $a \in \operatorname{Iann}(\mathcal{A})$. Take any $x . y \in \mathcal{A}$. Then we have

$$
\langle x a, y\rangle=\left\langle a, x^{l} y\right\rangle=\left\langle a y^{r}, x^{l}\right\rangle=0
$$

where $x^{l}$ is some left adjoint of $x$ and $y^{r}$ some right arljoint of $y$ (note that we don't need the uniqueness of the left and right adjoint in this moment ). This implies $a \in \operatorname{Rann}(\mathcal{A})$ and therefore $\operatorname{Lann}(\mathcal{A}) \subset \operatorname{Rann}(\mathcal{A})$. In a similar way we get $\operatorname{Rann}(\mathcal{A}) \subset \operatorname{Lann}(\mathcal{A})$ and therefore left and right annihilator of $\mathcal{A}$ are both equal to the annililator of $\mathcal{A}$ which is zcro by assumption.

(ii) Let $a^{l_{1}}$ and $a^{l_{2}}$ be two left adjoints of the clement $a$. Take any $x, y \in \mathcal{A}$. Then we have

$$
\left\langle x,\left(a^{l_{1}}-a^{l_{2}}\right) y\right\rangle=\left\langle. x, a^{l_{1}} y\right\rangle-\left\langle x, a^{l_{2}} y\right\rangle=\langle a \cdot r, y\rangle-\langle a x, y\rangle=0 .
$$

Using (i) we get $a^{l_{1}}=a^{l_{2}}=a^{l}$. In a similar way we can prove that the right adjoint is unique and

$$
a^{l l}=a^{r r}=a, \quad(\lambda a)^{r}=\bar{\lambda} a^{l}, \quad(\lambda a)^{r}=\bar{\lambda} a^{r}
$$

holds for all $a \in \mathcal{A}$ and all complex $\lambda$. $\Lambda$ lon we have for all $a, b, x, y \in \mathcal{A}$

$$
\begin{aligned}
& \left\langle\left((a b)^{l}-b^{l} a^{l}\right) \cdot x, y\right\rangle=\left\langle(a b)^{l} x, y\right\rangle-\left\langle b^{l} a^{l} x, y\right\rangle= \\
= & \langle x, a b y\rangle-\left\langle a^{l} \cdot x, b y\right\rangle=\langle\cdot x, a b y\rangle-\langle\cdot r, a b y\rangle=0 .
\end{aligned}
$$

(iii) The operators $L_{a}(x)=a x$ and $R_{a}(x)=x a$ both have adjoints (as operators acting on a Hilbert space $\mathcal{A}$ ) and are therefore continuous. By standard arguments we can now prove the continuity of the product.

(iv) Take any $a \in \mathcal{A}^{2 \perp}$. For all $x, y \in \mathcal{A}$ wc have

$$
\langle a \cdot r, y\rangle=\left\langle a, y x^{r}\right\rangle=0
$$

and using (i) we get $a=0$.

(v) An easy application of the closed graph theorem. 
(vi) First we take $y=a b$. 'Then wo have

$$
\begin{gathered}
\langle, r, y\rangle=\langle\cdot r, a b\rangle=\left\langle a^{l}, r, b\right\rangle= \\
=\left\langle a^{l}, b, r^{r}\right\rangle=\left\langle l^{l} a^{l}, r^{r}\right\rangle=\left\langle(a b)^{l}, . r^{r}\right\rangle=\left\langle y^{l}, x^{r}\right\rangle .
\end{gathered}
$$

By (iv), (v) and the contimuity of the inner product the alowe islentity hold for all $x, y \in \mathcal{A}$.

The following proposition contains an application of the Sakai's theorem and will be crucial in the proof of Theorem.

PROPOSITION. $\operatorname{Lrl} \mathcal{A}$ br as abore and $I): \mathcal{A} \longrightarrow \mathcal{A}$ a continuous deriration. The $n$ there exist continuous linear operators $T_{0}$ and $\zeta_{0}$ such that lhe following holds:

(i) $D=T_{0}-S_{0}$

(ii) $T_{0}(. r y)=T_{0}(x) y$ for all $r, y \in \mathcal{A}$,

(iii) $S_{0}(. x y)=x S_{0}(y)$ for all $x, y \in \mathcal{A}$.

(iv) $x T_{0}(y)=S_{0}(x) y$ for all $x, y \in \mathcal{A}$.

If $D$ is self-adjoint (as an oprrator acting on a Itillbry spars), $T_{0}$ and $S_{0}$ can also be taken selfadjoint.

REMARK. In the theory of $C^{*}$-algebras the pair $\left(T_{0}, S_{0}\right)$ is usually called a double centralizer.

PROOF. Define $L(\mathcal{A})$ and $R(\mathcal{A})$ to be the algebras of continuous left and right multipliers i.e.

$$
\begin{aligned}
& L(\mathcal{A})=\{T \in B(\mathcal{A}) ; T(x y)=T(x) y \text { for all } x, y \in \mathcal{A}\} \\
& R(\mathcal{A})=\{S \in B(\mathcal{A}) ; S(x y)=x, S(y) \text { for all } x, y \in \mathcal{A}\}
\end{aligned}
$$

Then $L(\mathcal{A})$ and $R(\mathcal{A})$ are von Neumann algebras and $L(\mathcal{A})^{\prime}=R(\mathcal{A})$ holds.

It is easy to see that $L(\mathcal{A})$ an $R(\mathcal{A})$ are algchras with identity. From Lemma(iii) we get that $L(\mathcal{A})$ and $R(\mathcal{A})$ are closed in the strong operator topology. We must see that they are also selfadjoint as subalgebras of $B(\mathcal{A})$. Take any contimunus left multiplier $T$ and compute

$$
\begin{aligned}
& \left\langle T^{*}(x y), z\right\rangle=\langle x y, T(z)\rangle=\left\langle. r, T(z) y^{r}\right\rangle= \\
= & \left\langle r, T\left(z y^{r}\right)\right\rangle=\left\langle T^{*}(x), z y^{r}\right\rangle=\langle T(x) y, z\rangle .
\end{aligned}
$$

Hence $T^{*}$ is a left multiplier and so $L(\mathcal{A})$ is a von Ncumann algebra. In a similar way we can prove that $R(\mathcal{A})$ is also a von Neumann algol,ra.

Take some continuous left multiplier $T$ and some continuous right multiplier $S$. By

$$
T S(x y)=T(x S(y))=T(x) S(y)=S(T(x) y)=S T(r y)
$$


and Lemma(iv), $T$ and $S$ commute. Thin shows that $I(\mathcal{A}) \subset I(\mathcal{A})^{\prime}$. To prove the converse observe that operators $L_{x}: y \mapsto x y$ are continuous left multiplier for all $x \in \mathcal{A}$. Take any $S \in L(\mathcal{A})^{\prime}$. Then

$$
S(x y)=S L_{r}(y)=L_{r} S(y)=x S(y)
$$

shows that $S$ is a right multiplier.

Now define $\Delta: \mathcal{L}(\mathcal{A}) \longrightarrow \mathcal{L}(\mathcal{A})$ by $\Delta(T)=D T-T D$. Wre must show that $\Delta(T)$ is in fact left multiplier:

$$
\begin{gathered}
\Delta(T)(x y)=D T(. x y)-T I)(x y)=D(T(. x) y)-T(D(x) y+x I(y))= \\
=(D T)(x) y+T(. x) D(y)-(T D)(. x) y-T(. r) D(y)=\Delta(T)(. r) y .
\end{gathered}
$$

Straightforward verification shows that $\Delta$ is indeed a derivation. Now we nee a well-known result that every derivation of a von Neumann algebra is inner. There exists a left multiplier $T_{0}$ such that

$$
\Delta(T)=T_{0} T-T T_{0}=D T-T D .
$$

Thus for all $T \in \mathcal{L}(\mathcal{A})$ we have

$$
\left(T_{0}-D\right) T=T\left(T_{0}-D\right) .
$$

So $S_{0}=T_{0}-D \in \mathcal{L}(\mathcal{A})^{\prime}=\mathcal{R}(\mathcal{A})$. We must sce that $x T_{0}(y)=S_{0}(x) y$ holds for all $x, y \in \mathcal{A}$ :

$$
\begin{gathered}
x T_{0}(y)-S_{0}(x) y=x T_{0}(y)-x S_{0}(y)+x S_{0}(y)-T_{0}(x) y+T_{0}(x) y-S_{0}(x) y= \\
=x D(y)+S_{0}(x y)-T_{0}(x y)+D(x) y=x D(y)-D(x y)+D(x) y=0 .
\end{gathered}
$$

Finally suppose that $D$ is sclf-adjoint. From the fact that left and right multipliers are closed for adjoints (the second paragraph of this proof), it follows that

$$
D=D^{*}=\frac{1}{2}\left(T_{0}+T_{0}^{*}\right)-\frac{1}{2}\left(S_{0}+S_{0}^{*}\right)
$$

is a self-adjoint decomposition with properties (i)-(iv).

Now we are ready for the final step.

PROOF OF THE TIIEOREM. Define a linear operator $\Phi$ on $\mathcal{A}$ with $\Phi(x)=\left(x^{r}\right)^{l}$. By Lemma(v) $\Phi$ is continuous and by Lemma(iii) $\Phi$ is an automorphism of $\mathcal{A}$. Using Iemma(vi) we obtain

$$
\langle\Phi(x), x\rangle=\left\langle x^{r l}, x\right\rangle=\left\langle x^{r}, x^{r l l}\right\rangle=\left\|r^{r}\right\|^{2} \geq 0
$$

which shows that $\Phi$ is sclfadjoint and that its spectrum is contained in $\mathbb{R}^{+}$. By [2, Theorem 15 , page 91] $\Phi=\exp (D)$ for some continuous (obviously self-adjoint) derivation $D$ of $\mathcal{A}$.

According to the Proposition, we may decompose $D=T_{0}-S_{0}$. Since $D$ is sclfadjoint, we can also choose $T_{0}$ and $S_{0}$ both selfadjoint. Define $U=\exp \left(T_{0}\right)$ and $V=\exp \left(S_{0}\right)$. Then $U$ and $V$ are 
positive invertible operators and $\Phi=I^{\prime} V^{-1}$ holds. $I^{r}$ is a left multiplier, $V$ is a right multiplier and therefore they commute. It is easy to ser that $x I^{\prime}(y)=I^{\prime}(r) y$ holds for all $x, y \in \mathcal{A}$ (compare Proposition(iv) ).

Now define $\langle x, y\rangle_{1}=\langle U(x), y\rangle$. It remains to verify that $\left(\mathcal{A},\langle,\rangle_{1}, r\right)$ is an $H^{*}$-algebra. This is done in the following computation:

$$
\begin{gathered}
\left\langle x, z y^{r}\right\rangle_{1}=\left\langle I^{r}(x), z y^{r}\right\rangle=\left\langle I^{r}(x) y, z\right\rangle= \\
=\left\langle I^{r}(r y), z\right\rangle=\langle. r y, z\rangle_{1}, \\
\left\langle y, x^{r} z\right\rangle_{1}=\left\langle I^{\prime}(y), x^{r} z\right\rangle=\left\langle r^{r l} I^{r}(y), z\right\rangle= \\
-\langle\Phi(x) U(y), z\rangle=\left\langle I^{\prime} I^{-1}(x) U(y), z\right\rangle=\left\langle I^{\prime} I^{r} V^{-1}(x) y, z\right\rangle= \\
=\left\langle U^{\prime}(x) y, z\right\rangle=\langle U(x y), z\rangle=\langle. r y, z\rangle_{1} .
\end{gathered}
$$

The proof is thus completed.

REMARK. It is not known whether the nonassociative $I I^{*}$-algebras can be characterized in the same way. Saworotnow's original proof is entirely associative. In our proof the associativity is used once in the proof of the Proposition namely when we proved that $L(\mathcal{A})$ is a commutant of $R(\mathcal{A})$. This is true if and only if $\mathcal{A}$ is associative.

\section{REFERENCFS}

1. W. AMBROSE, Structure theorems for a special class of Banach algebras, Trans. Almer Mlath. $\underline{\text { Soc. }} \underline{57}$ (1945), 364-386.

2. F.F. BONSAL and J. DUNCAN, Complete normed algehras, Frgeb. Math. Grenzgeb. 80 (1973)

3. M. CABRerA, J. MARTineZ and A. RODRIGUeZ, Nonassocialive real $H^{*}$-algebras, Publ. Mat. $\underline{32}$ (1988), 267-274

4. M. CABrera, J. MARTineZ and A. RODriciefZ, Mal'cev $I^{*}$-algebras, Malli. Proc Camb. Phil. Soc. 103 (1988), 463-471

5. M. CAHIRERA, J. MARTINEZ and A. RODRIGLEZ, Structurable $H^{*}$-algebras, J. Algebra 117 (1992), 19-62

6. J.A. CUENCA, A. GARCIA and C. MARTIN, Structure theory for $L^{*}$-algebras, Math. Proc. Camb. Phil. Soc. 107 (1990), 361-365

7. S. SAKAI, Derivations of $W^{*}$-algebras, Ann. Math. $\underline{83}$ (1966), 273-279

8. P.P. SAWOROTNOW, On generalization of notition of $H^{*}$-algchra, Proc. Amer. lath. Soc. $\underline{8}$ (1957), 56-62

9. P.P. SAWOROTNOW, On two-sided $I^{*}$-algebras, Par. J. Math 16 (1966), 365-370

10. P.P. SAWOROTNOW, A generalized Hilbert space, Duke Math. J $\underline{35}$ (1968), 191-197

11. P.P. SAWOROTNOW, Representation of a complemented algehra on a locally compact space, Illinois $\mathrm{J}$. Math. 14 (1970), 674-677

12. J.F. SMITH and P.P. SAWOROTNOW, On some classes of scalar-product algebras, Pac. J. Math. 11 (1961), 739-750

13. B. ZALAR, Continuity of derivations on Mal'cev $H^{*}$-algehras, Math. Proc. Camb. Phil. Soc. $\underline{110}$ (1991), 455-459 


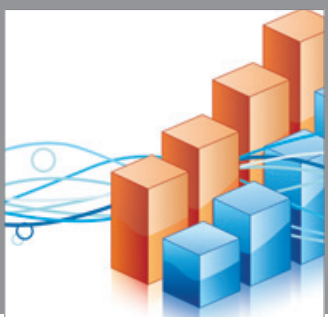

Advances in

Operations Research

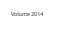

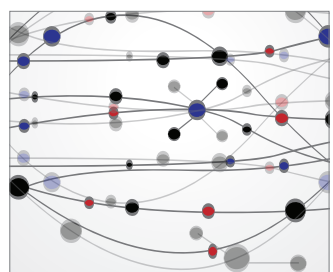

\section{The Scientific} World Journal
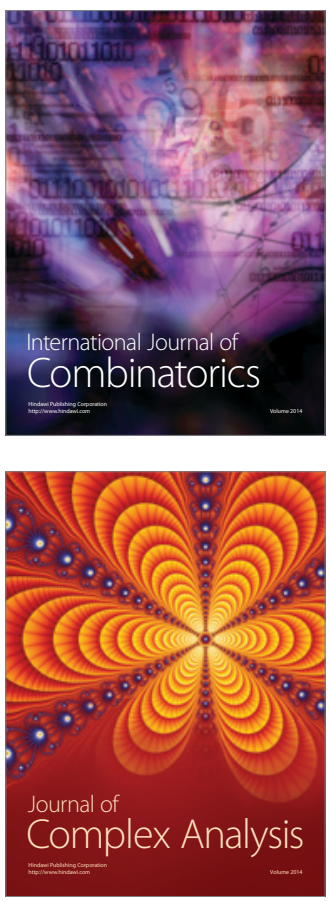

International Journal of

Mathematics and

Mathematical

Sciences
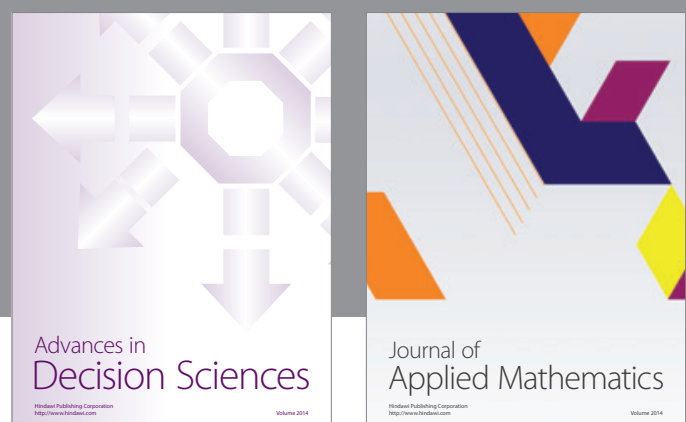

Journal of

Applied Mathematics
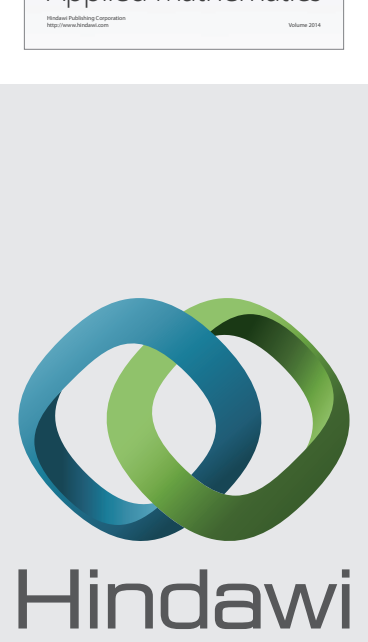

Submit your manuscripts at http://www.hindawi.com
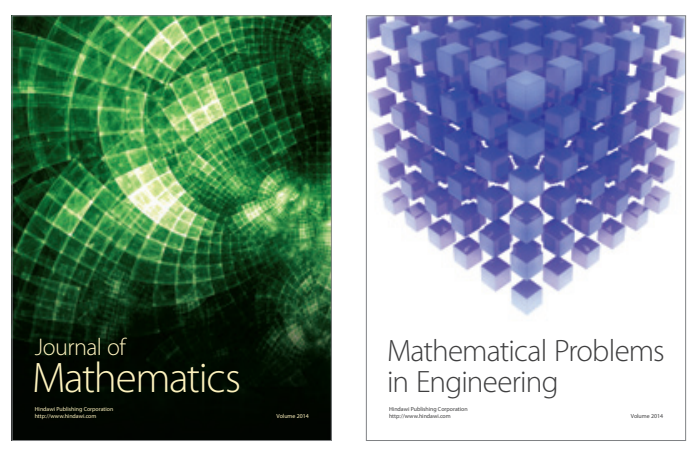

Mathematical Problems in Engineering
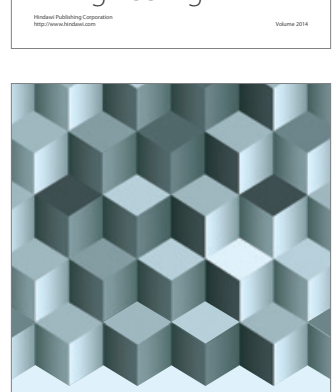

Journal of

Function Spaces
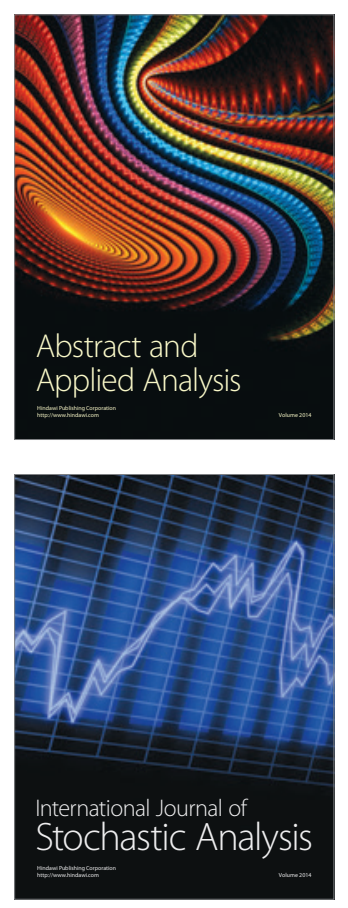

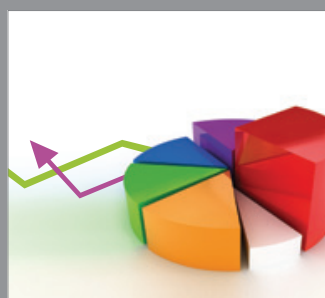

ournal of

Probability and Statistics

Promensencen
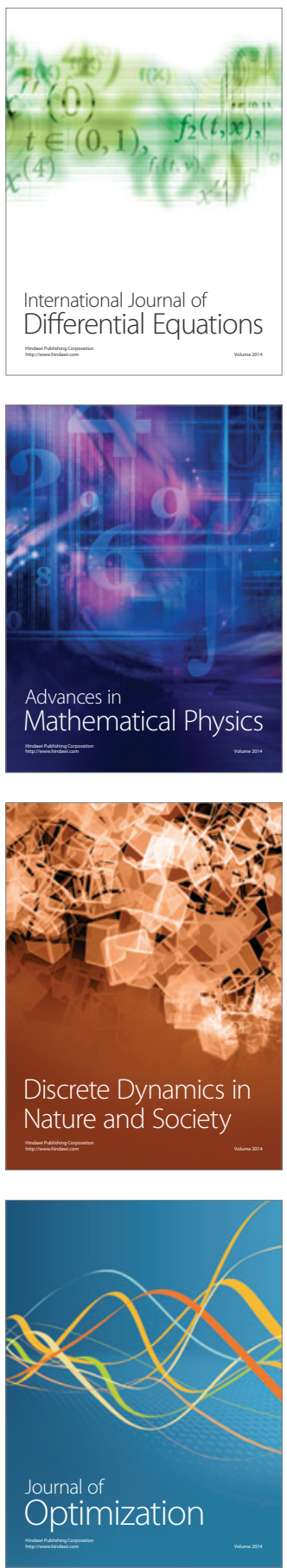